\title{
MicroRNA-146a/Toll-like receptor 4 signaling protects against severe burn-induced remote acute lung injury in rats via anti-inflammation
}

\author{
JINHUA LUO, JIANHUA ZHAN, HAOYUAN YOU and XING CHENG
}

Department of Burns, The First Affiliated Hospital of Nanchang University, Nanchang, Jiangxi 330006, P.R. China

Received August 9, 2017; Accepted December 14, 2017

DOI: $10.3892 / \mathrm{mmr} .2018 .8877$

\begin{abstract}
The present study investigated the preventive effects of microRNA (miR)-146a against severe burn-induced remote acute lung injury (ALI) in rats and the underlying mechanism. The surface area of the skin was immersed in $100^{\circ} \mathrm{C}$ water for 5-10 sec on the dorsal surface. The expression level of miR-146a was significantly downregulated in rats with burn-induced ALI. Downregulation of miR-146a increased inflammation, and inducible nitric oxide synthase (iNOS) and cyclooxygenase-2 (COX-2) expression in a model of ALI in vitro via the promotion of the Toll-like receptor (TLR)4/nuclear factor $(\mathrm{NF})-\kappa \mathrm{B}$ signaling pathway. In addition, the overexpression of miR-146a reduced inflammation, and iNOS and COX-2 protein expression in the model of ALI in vitro via the suppression of the TLR4/NF- $\kappa \mathrm{B}$ signaling pathway. A TLR4 inhibitor reduced the function of anti-miR-146a on inflammation in the model of ALI. Collectively, the results of the present study demonstrated the preventive effects of miR-146a against severe burn-induced remote ALI in rats through the anti-inflammatory-regulated TLR4/NF- $\mathrm{KB}$ signaling pathway.
\end{abstract}

\section{Introduction}

Severe burn-induced remote acute lung injury (ALI) is a universal issue challenging clinical intensive treatment (1). Severe burns have been reported to induce marked alterations to the organic internal environment. Factors including traumatic stress, direct injuries of heating power and hypoperfusion may induce the upregulation of expression levels of pro-inflammatory cytokines (2). In addition, these factors may activate a variety of inflammatory cells, including mononuclear macrophages, neutrophil granulocy tes, lymphocytes, platelets and endothelial cells $(3,4)$. Furthermore,

Correspondence to: Dr Jianhua Zhan, Department of Burns, The First Affiliated Hospital of Nanchang University, 17 Yongwaizheng Street, Nanchang, Jiangxi 330006, P.R. China

E-mail: zhanjujuz@163.com

Key words: microRNA-146a, acute lung injury, Toll-like receptor 4, nuclear factor- $\kappa \mathrm{B}$ adhesion molecules of vascular endothelial cells (VECs) and while blood cells (WBCs) have been reported to be upregulated $(3,4)$. The activated WBCs may then induce a respiratory burst and degranulation as the adhesion intensifies. As a result, metabolites and proinflammatory cytokines, including fibrinolysin, active oxygen and arachidonic acid, would be released. This then causes broader damage to VECs and other tissues (5).

Severe burn-induced remote ALI is characterized by the pathological features of a uncontrolled inflammatory response, and it may broadly damage pulmonary VECs and alveolar epithelial cells (4). Severe-burn induced remote ALI has been reported to exhibit morbidity and mortality rates of $20-40 \%$ (4). However, no treatments are currently available within intensive care units (4). Uncontrollable inflammatory mediators are closely associated with ALI (4). Macrophages were in patients with ALI to induce inflammation (6). In addition, numerous inflammatory cytokines are released, including interleukin (IL)-6, IL-18, tumor necrosis factor- $\alpha$ (TNF- $\alpha$ ) and interferon- $\gamma$. Furthermore, macrophages, neutrophils, granulocytes, lymphocytes and endothelial cells are activated; oxygen radicals, proteolytic enzymes and arachidonic acid are also released (4). Inflammatory responses within the lungs may induce injury to the alveolar capillary membrane. Subsequently, the ALI is initiated and the inflammatory response is induced (7).

Alterations in microRNA (miR) expression have been associated with immune responses, the inflammatory signaling pathway and the pathogenesis of inflammatory pulmonary diseases, such as ALI (8). Therefore, miR may be promising novel therapeutic targets. miR-induced alterations in the expression of associated genes are generally moderate (9); however, its results may affect the expression of several genes and numerous biological processes (10). Therefore, miR may be considered as a potential marker of ALI. The protective effects of miR-146a on severe burn-induced remote ALI in rats were examined in the present study. The results suggested that miR-146a protected against severe burn-induced ALI via the anti-inflammatory pathway.

\section{Materials and methods}

Animals. All experimental protocols were undertaken in accordance with the Guide for the Care and Use of Laboratory 
Animals by the National Institutes of Health (11), with the approval of the Animal Experimental Ethics Committee of the First Affiliated Hospital of Nanchang University (Nanchang, China). Healthy male Sprague-Dawley rats (200-250 g, n=12) were purchased from Laboratory Animal Center of the Medical Department of Nanchang University (Nanchang, China) and used in the present study. The animals were housed at $22-24^{\circ} \mathrm{C}$, 55-60\% humidity, $0.038 \% \mathrm{CO}_{2}, 12 \mathrm{~h}$ light/dark cycle and fed a standard animal diet with food and tap water ad libitum.

Experimental design and burn procedure. The rats were randomly divided into three groups, each containing 10 rats: Sham-operated group, ALI group and oleuropein-treated group. The rats of the ALI and oleuropein-treated groups were anesthetized with $30 \mathrm{mg} / \mathrm{kg}$ of pentobarbital sodium Sigma-Aldrich (Merck KGaA, Darmstadt, Germany) and shaved on the dorsal and lateral surfaces. The surface area of the skin was immersed in $100^{\circ} \mathrm{C}$ water for $5-10 \mathrm{sec}$ on the dorsal surface. The skin of all rats were quickly dried, total body surface area was $30 \%$ at all skin area. Sham-operated rats were anesthetized with $30 \mathrm{mg} / \mathrm{kg}$ of pentobarbital and shaved on the dorsal and lateral surfaces.

$R N A$ isolation and reverse transcription-quantitative polymerase chain reaction ( $R T-q P C R)$. Total RNA was extracted using TRIzol ${ }^{\circledR}$ (Thermo Fisher Scientific, Inc., Waltham, MA, USA) from lung tissue samples or cells and $\sim 1 \mu \mathrm{g}$ total RNA was then used to produce cDNA using TaqMan ${ }^{\circledR}$ MicroRNA Reverse Transcription kit (Thermo Fisher Scientific, Inc.) at $37^{\circ} \mathrm{C}$ for $60 \mathrm{~min}$ and $85^{\circ} \mathrm{C}$ for $1 \mathrm{~min}$ by a TaqMan $7900(\mathrm{ABI})$ real-time PCR machine (Invitrogen; Thermo Fisher Scientific, Inc.). miR-146a, inducible nitric oxide synthase (iNOS) and cyclooxygenase-2 (COX-2) mRNA expression levels were determined by RT-qPCR using TransStart ${ }^{\mathrm{TM}}$ SYBR-Green qPCR Supermix (Beijing Transgen Biotech Co., Ltd., Beijing, China). The following thermocycling conditions were used for the PCR: Initial denaturation at $95^{\circ} \mathrm{C}$ for $10 \mathrm{~min}$; 40 cycles of $95^{\circ} \mathrm{C}$ for $15 \mathrm{sec}, 60^{\circ} \mathrm{C}$ for $30 \mathrm{sec}$ and $72^{\circ} \mathrm{C}$ for $30 \mathrm{sec}$. The primer sequences used were as follows: miR-146a forward, 5'-TGAGAACTGAATTCCATGGGTT-3' and reverse, 5'-TCA CCCGTAGAACCGACCTT-3'; iNOS forward, 5'-CCCTTC CGAAGTTTCTGGCAGCAG-3' and reverse, 5'-GGCTGT CAGAGCCTCGTGGCTTTG-3'; COX-2 forward, 5'-ATG CTCCTGCTTGAGTATGT-3' and reverse, 5'-CACTACATC CTGACCCACTT-3'; GAPDH forward, 5'-AACTTTGGCATT GTGGAAGG-3' and reverse, 5'-ACACATTGGGGGTAG GAACA-3'; and U6 forward, 5'-ATTGGAACGATACAGAGA AGATT-3' and reverse, 5'-GGAACGCTTCACGAATTTG-3'. The relative levels of the target genes were normalized using the $2^{-\Delta \Delta \mathrm{Cq}}$ method (12).

Cell culture and transfection. 16HBE cells (human bronchial epithelial) were obtained from the Cell Bank of Type Culture Collection of Chinese Academy of Sciences (Shanghai, China) and maintained in RPMI-1640 (Gibco; Thermo Fisher Scientific, Inc.) supplemented with $10 \%$ fetal bovine serum (Gibco; Thermo Fisher Scientific, Inc.) in $5 \% \mathrm{CO}_{2}$ and $95 \% \mathrm{O}_{2}$ at $37^{\circ} \mathrm{C}$. miR-146a (5'-UGAGAACUGAAUUCCAUG GGUU-3' and 5'-CCCAUGGAAUUCAGUUCUCAUU-3'), anti-miR-146a (5'-AACCCAUGGAAUUCAGUUCUCA-3' and

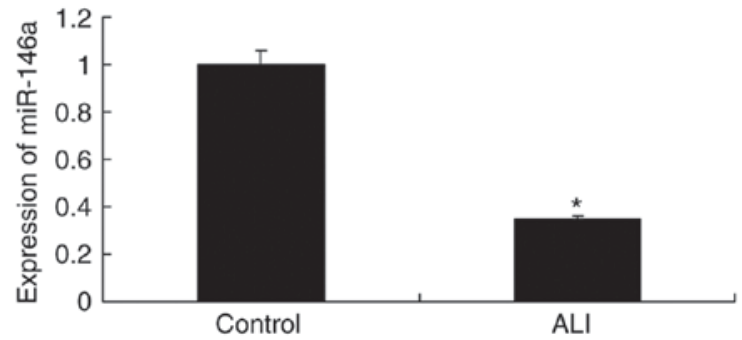

Figure 1. Expression of miR-146a is decreased in burn-induced ALI. ${ }^{*} \mathrm{P}<0.01$ vs. control group. ALI, acute lung injury; miR, microRNA.

5'-GCTGTCAACGATACGCTACGTAACG-3'), and negative mimics (5'-CCCCCCCCCCCCC-3' and 5'-CCCCCCCCCCC CCC-3') were obtained from Sangon Biotech Co., Ltd., (Shanghai, China). 16HBE cells were transfected with $200 \mathrm{ng}$ of miR-146a, anti-miR-146a or negative mimics using Lipofectamine $^{\circledR} 2000$ reagent (Invitrogen; Thermo Fisher Scientific, Inc.). Following transfection for $48 \mathrm{~h}$, ALI was induced within 16HBE cells using lipopolysaccharide (LPS; $100 \mathrm{ng} / \mathrm{ml}$; Beyotime Institute of Biotechnology, Nanjing, China) for $6 \mathrm{~h}$. Control, $16 \mathrm{HBE}$ cells were transfected with negative control mimics for $48 \mathrm{~h}$ and treated with LPS $(100 \mathrm{ng} / \mathrm{ml})$ and TAK-242 (2 nM; MedChemExpress, Monmouth Junction, NJ, USA) for $6 \mathrm{~h}$.

Measurement of nuclear factor $(N F)-\kappa B, I L-6, I L-10$ and $T N F-\alpha$. Bronchoalveolar lavage samples were collected and centrifuged at $12,000 \mathrm{x}$ for $10 \mathrm{~min}$ at $4^{\circ} \mathrm{C}$. The supernatant fluids were used to measure NF- $\kappa \mathrm{B}$ (cat. no. H202), IL-6 (cat. no. H007), IL-10 (cat. no. H009) and TNF- $\alpha$ (cat. no. H052) levels using ELISA kits (Nanjing Jiancheng Bioengineering Institute, Nanjing, China) according to the manufacturer's protocols.

Western blot analysis. Protein $(50 \mu \mathrm{g})$ was excised from lung tissue using homogenated extraction buffer on ice and centrifuged at $12,000 \mathrm{x}$ g for $10 \mathrm{~min}$ at $4^{\circ} \mathrm{C}$ in radioimmunoprecipitation assay (Sangon Biotech Co., Ltd.). Protein concentrations were determined using a Bicinchoninic Acid protein assay reagent (Sangon Biotech Co., Ltd.). Protein extracts $(50 \mu \mathrm{g})$ were separated via $10 \%$ SDS-PAGE (Sangon Biotech Co., Ltd.) and electrotransferred onto a nitrocellulose membrane. The membranes were blocked with $5 \%$ skimmed milk in TBS with $0.1 \%$ Tween-20 for $1 \mathrm{~h}$ at $37^{\circ} \mathrm{C}$ and immunoblotted with primary antibodies specific for NF-кB (cat. no. sc-109, 1:3,000; Santa Cruz Biotechnology, Inc., Dallas, TX, USA), Toll-like receptor 4 (cat. no. sc-10741, TLR4; 1:3,000; Santa Cruz Biotechnology, Inc.) and GAPDH (cat. no. sc-25778, 1:4,000; Santa Cruz Biotechnology, Inc.) overnight at $4^{\circ} \mathrm{C}$. Following three washes with Tris-buffered saline- $0.1 \%$ Tween-20, the membrane was incubated with anti-rabbit immunoglobulin $\mathrm{G}(\mathrm{H}+\mathrm{L})$, Biotinylated secondary antibody (cat. no. 14708, 1:5,000; Cell Signaling Technology, Inc., Danvers, MA, USA) for $1 \mathrm{~h}$ at $37^{\circ} \mathrm{C}$ and detected using an enhanced chemiluminescence plus detection kit (Amersham; GE Healthcare, Chicago, IL, USA) and exposed to film and analyzed using Image Lab 3.0 (Bio-Rad Laboratories, Inc., Hercules, CA, USA). 
A
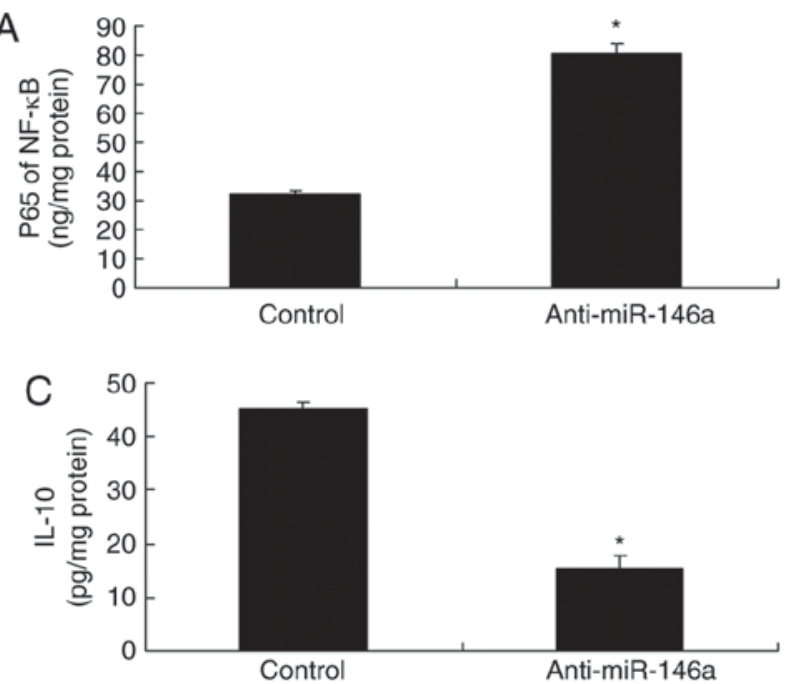

B
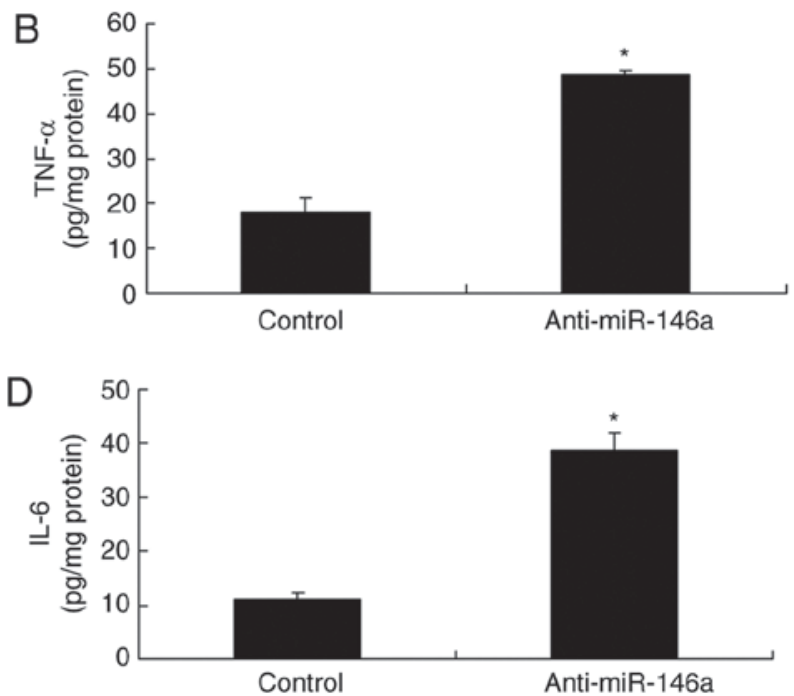

Figure 2. Downregulation of miR-146a with anti-miR-146a increases inflammation in a model of acute lung injury. (A) NF- $\mathrm{B}$, (B) TNF- $\alpha$, (C) IL-10 and (D) IL-6 expression levels. ${ }^{*} \mathrm{P}<0.01$ vs. control group. miR, microRNA; NF- $\mathrm{B}$, nuclear factor- $\kappa \mathrm{B}$; TNF- $\alpha$, tumor necrosis factor- $\alpha$; IL, interleukin.

Statistical analysis. All data were expressed as the mean \pm standard error of the mean using SPSS 17.0 (SPSS, Inc., Chicago, IL, USA). The results were analyzed statistically using one-way analysis of variance and Duncan's multiple range test. $\mathrm{P}<0.05$ was considered to indicate a statistically significant difference.

\section{Results}

Expression of miR-146a in burn-induced ALI rat. As presented in Fig. 1, the expression levels of miR-146a were significantly downregulated in the rat model of burn-induced ALI compared with the levels in the control group.

Downregulation of $\mathrm{miR}-146$ a increases inflammation in a model of ALI in $16 H B E$ cells. Subsequently, the function of miR-146a in ALI was explored and miR-146a expression was inhibited using anti-miR-146a mimics. Levels of $\mathrm{NF}-\kappa \mathrm{B}$, IL-6, IL-10 and TNF- $\alpha$ were measured using ELISA kits. As demonstrated in Fig. 2, NF- $\kappa$ B, IL- 6 and TNF- $\alpha$ levels within the model of ALI were significantly higher in response to miR-146a downregulation than those in the control group. IL-10 expression levels within the ALI model were significantly reduced in response to miR-146a downregulation compared with those in the control group.

Downregulation of miR-146a increases iNOS and COX-2 mRNA expression levels in a model of ALI in 16HBE cells. Downregulation of miR-146a significantly induced iNOS and COX-2 mRNA expression within the model of ALI compared with the levels in the control group (Fig. 3). There results demonstrated that anti-miR-146a regulates iNOS and COX-2 expression to affect the development of ALI.

Downregulation of miR-146a upregulates the TLR4/NF- $\mathrm{BB}$ signaling pathway in a model of ALI in $16 H B E$ cells. The mechanism of the effect of miR-146a on inflammation within the model of ALI was investigated by analyzing TLR4 and $\mathrm{NF}-\kappa \mathrm{B}$ protein expression levels. As presented in Fig. 4, the downregulation of miR-146a significantly increased TLR4 and $\mathrm{NF}-\kappa \mathrm{B}$ protein expression levels within the ALI model compared with the levels in the control group.

Upregulation of miR-146a decreases inflammation in a model of ALI in 16HBE cells. miR-146a expression levels were upregulated to investigate the mechanism of miR-146a on inflammation within the model of ALI. As demonstrated in Fig. 5, upregulation of miR-146a significantly decreased NF- $\kappa$ B, IL- 6 and TNF- $\alpha$ expression levels, and increased IL-10 expression levels within the model of ALI compared with the levels in the control group.

Upregulation of miR-146a decreases iNOS and COX-2 mRNA expression levels in an ALI model in $16 \mathrm{HBE}$ cells. iNOS and COX-2 mRNA expression levels in the ALI model were significantly reduced by the increased expression of miR-146a compared with the level in the control group (Fig. 6). Therefore, miR-146a reduced the expression of iNOS and COX-2 expression to ameliorate ALI.

Upregulation of miR-146a suppresses the TLR4/NF- $\kappa B$ signaling pathway within a model of ALI in $16 \mathrm{HBE}$ cells. TLR4 and NF- $\mathrm{B}$ protein expression levels were significantly suppressed within the model of ALI following miR-146a upregulation compared with the levels in the control group (Fig. 7).

TLR4 inhibitor reduces the function of anti-miR-146a on the TLR4/NF- $\kappa B$ signaling pathway within a model of ALI in $16 H B E$ cells. In order to confirm the role of the TLR4/NF- $\kappa B$ signaling pathway in the effect of miR-146a on ALI, TAK-242 inhibitor was employed to reduce TLR4 and NF- $\kappa \mathrm{B}$ protein expression. The TLR4 inhibitor suppressed TLR4 and NF- $\kappa \mathrm{B}$ protein expression levels within the model of ALI following anti-miR-146a treatment, compared with in the levels observed in the anti-miR-146a group (Fig. 8).

TLR4 inhibitor reduces the function of anti-miR-146a on inflammation in a model of ALI in 16HBE cells. The induction 



Figure 3. Downregulation of miR-146a with anti-miR-146a increases iNOS and COX-2 mRNA expression in a model of acute lung injury. (A) iNOS and (B) COX-2 mRNA expression levels. " $\mathrm{P}<0.01$ vs. control group. miR, microRNA; iNOS, inducible nitric oxide synthase; COX-2, cyclooxygenase-2.

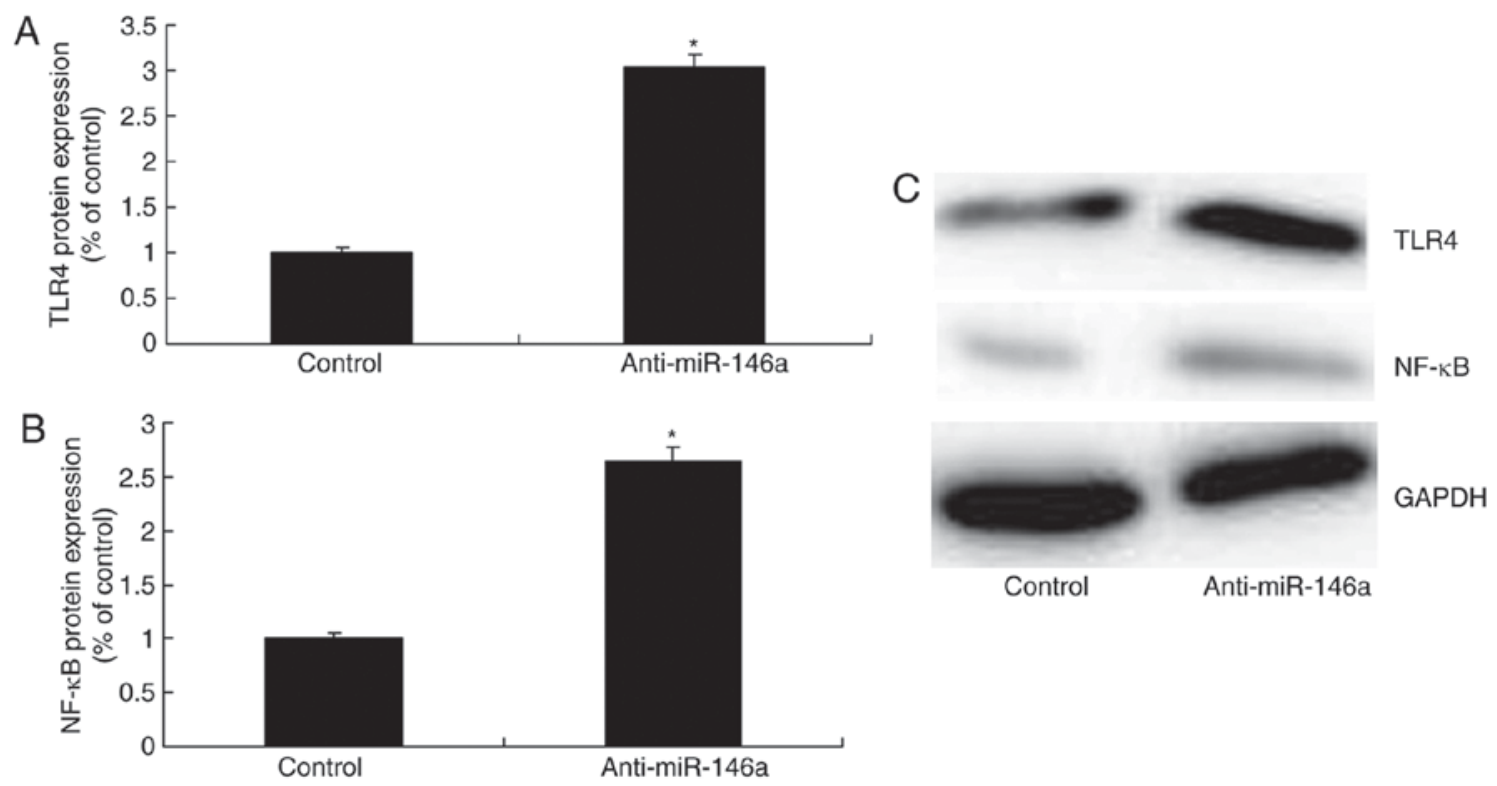

Figure 4. Downregulation of miR-146a with anti-miR-146a induces the TLR4/NF- $\mathrm{kB}$ signaling pathway in a model of acute lung injury. Quantified protein

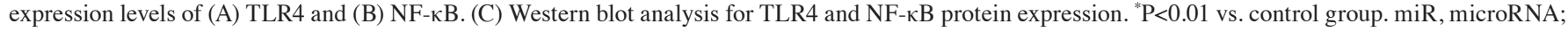

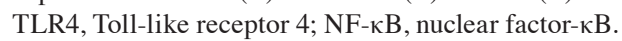
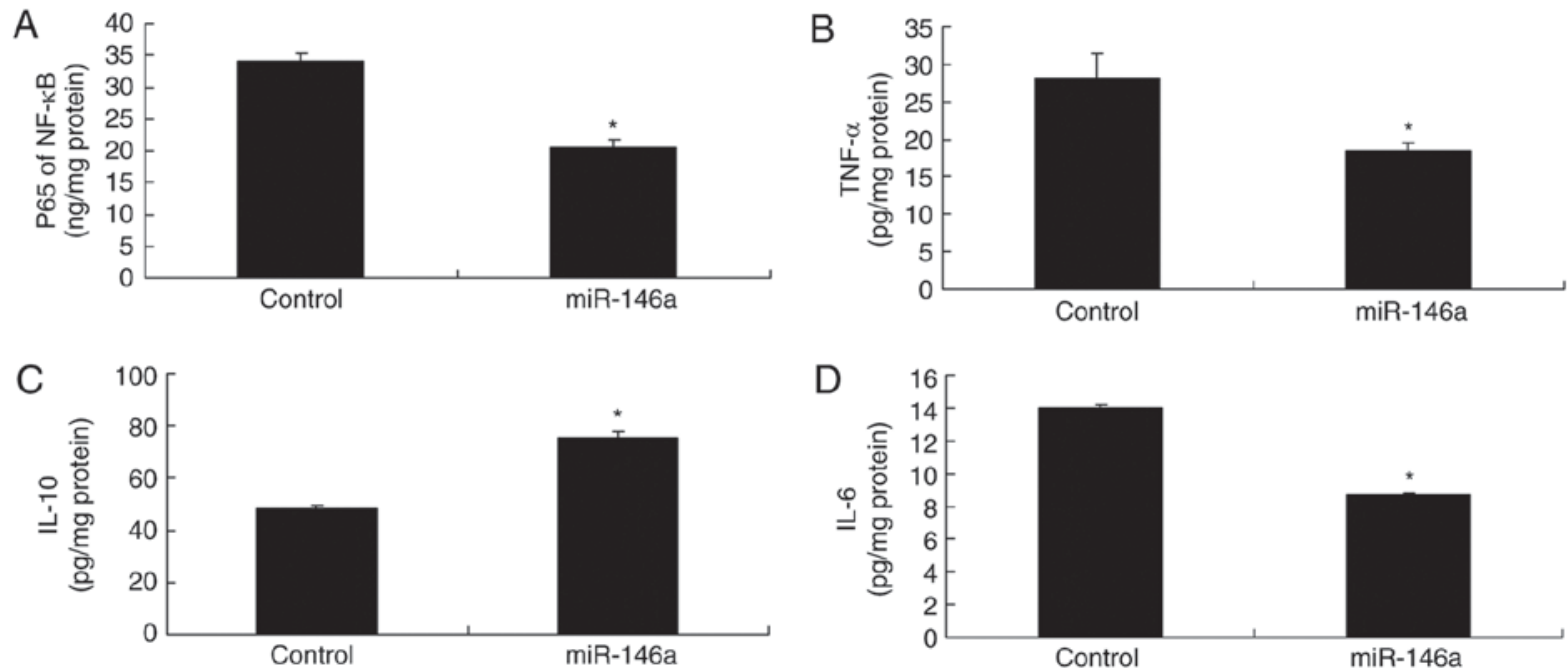

Figure 5. Upregulation of miR-146a decreases inflammation in a model of acute lung injury. (A) NF-kB, (B) TNF- $\alpha$, (C) IL-10 and (D) IL-6 protein expression

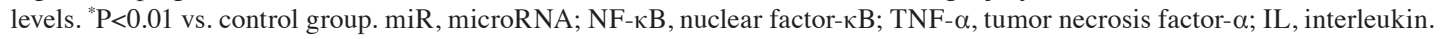

of NF- $\kappa B$, IL-6 and TNF- $\alpha$ levels, and inhibition of IL-10 levels within the model of ALI following anti-miR-146a treatment were significantly reversed by the inhibition of TLR4, compared with in the levels in the anti-miR-146a group (Fig. 9). 

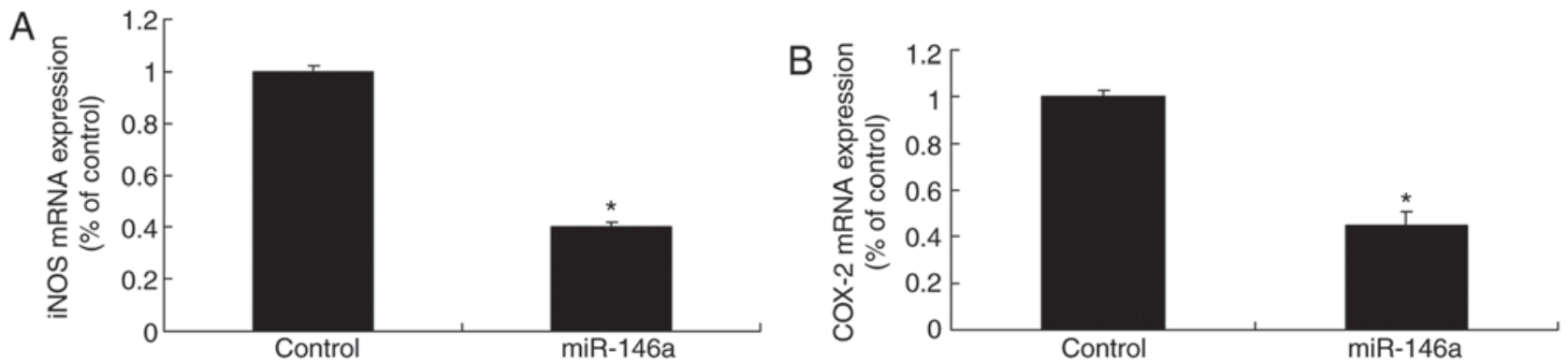

Figure 6. Upregulation of miR-146a decreases iNOS and COX-2 mRNA expression in a model of acute lung injury. (A) iNOS and (B) COX-2 mRNA expression levels. "P<0.01 vs. control group. miR, microRNA; iNOS, inducible nitric oxide synthase; COX-2, cyclooxygenase-2.
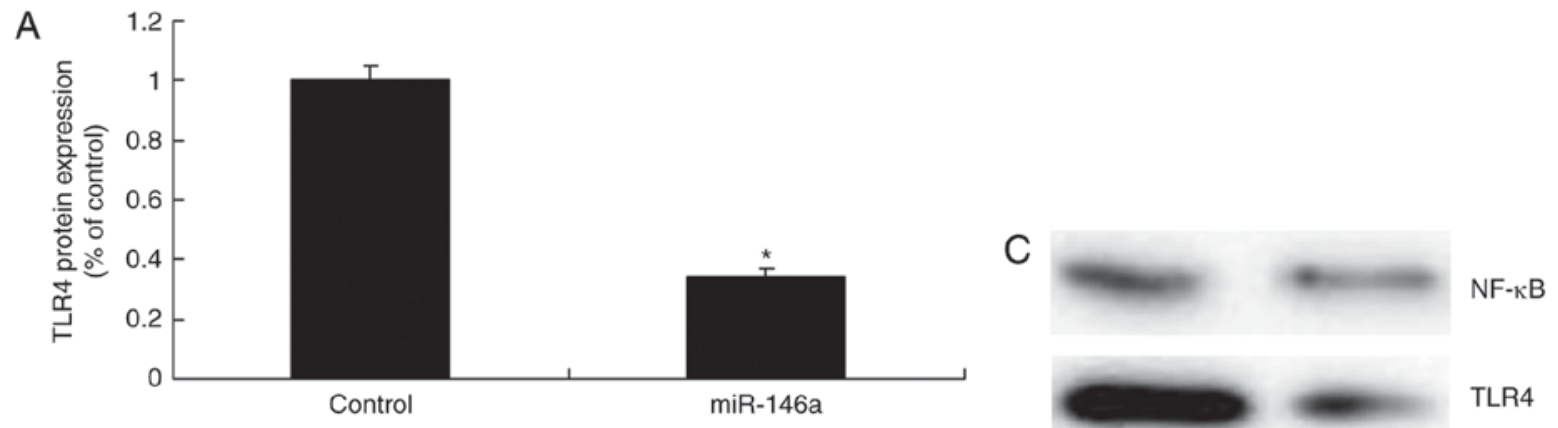

B


Figure 7. Upregulation of miR-146a suppresses the TLR4/NF- $\mathrm{KB}$ signaling pathway in a model of acute lung injury. Quantified protein expression levels of

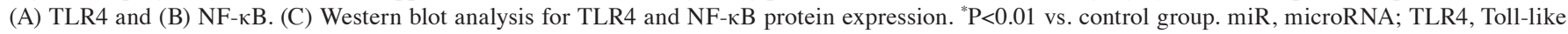



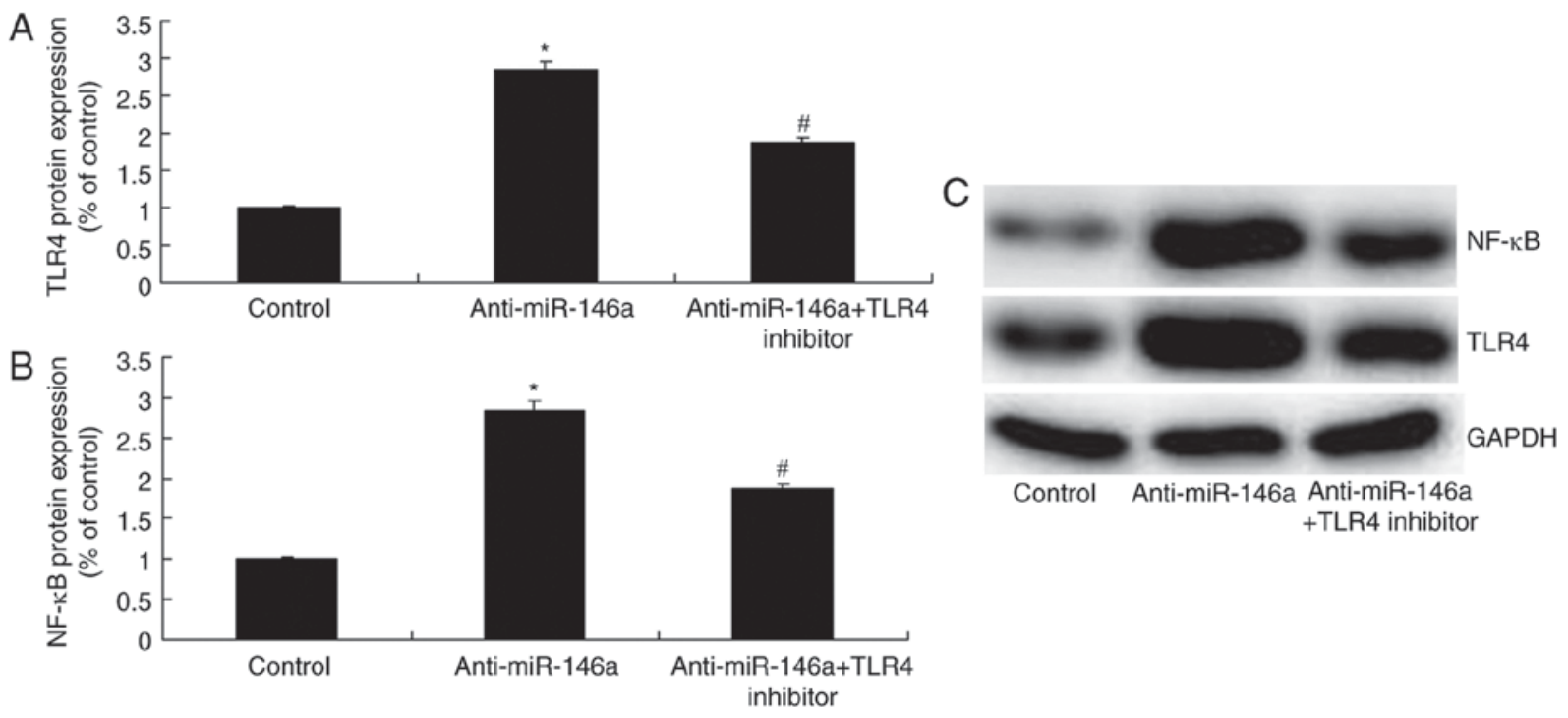

Figure 8. TLR4 inhibitor reduces the function of anti-miR-146a on the TLR4/NF- $\mathrm{B}$ B signaling pathway in a model of acute lung injury. Quantified protein

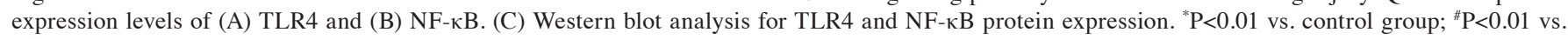

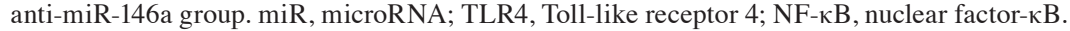



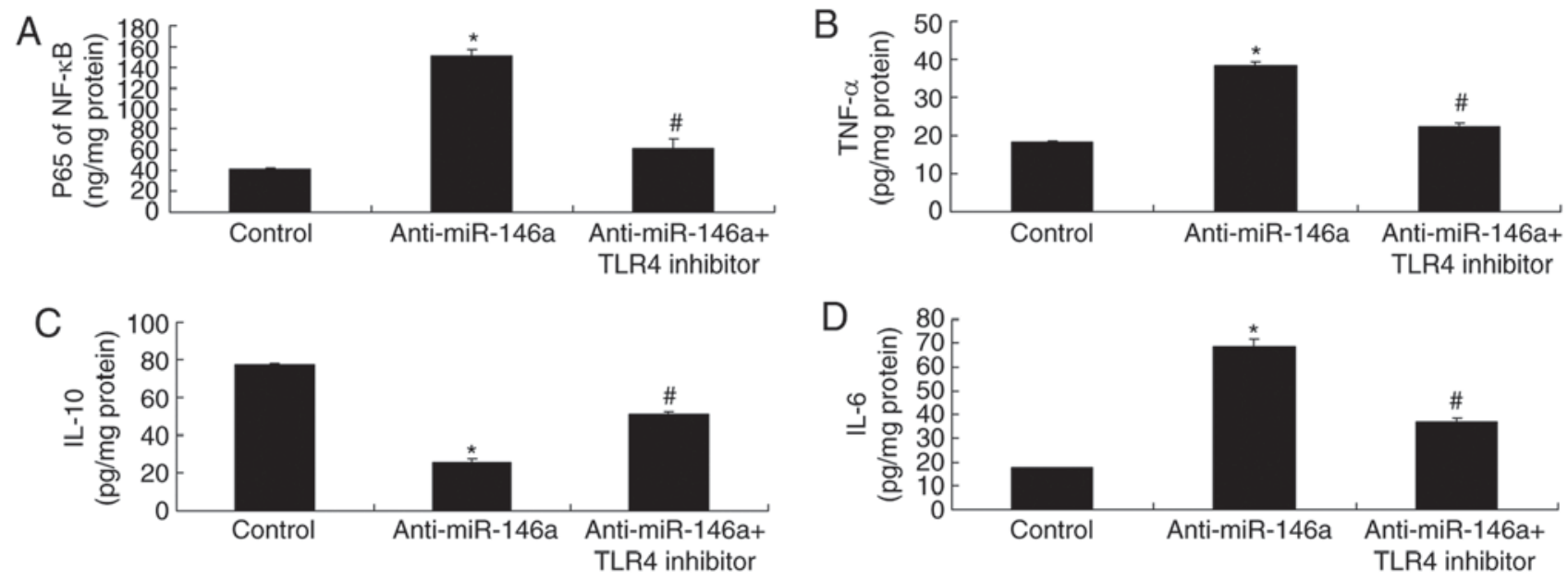

Figure 9. TLR4 inhibitor reduces the function of anti-miR-146a on inflammation in a model of acute lung injury. (A) NF- $\kappa B$, (B) TNF- $\alpha$, (C) IL-10 and (D) IL-6

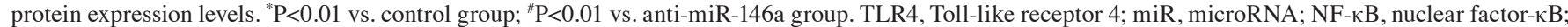
TNF- $\alpha$, tumor necrosis factor- $\alpha$; IL, interleukin.
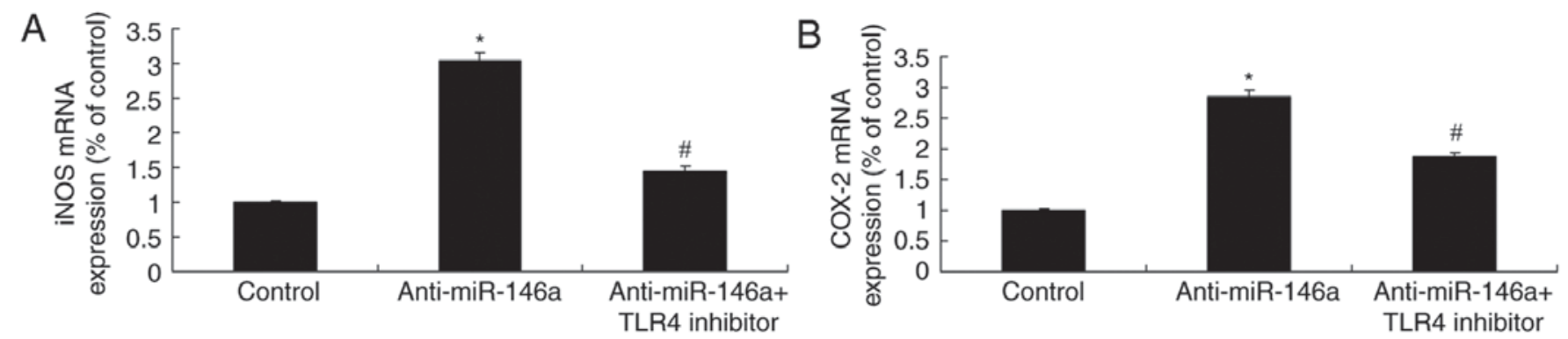

Figure 10. TLR4 inhibitor reduces the function of anti-miR-146a on iNOS and COX-2 mRNA expression in a model of acute lung injury. (A) iNOS and (B) COX-2 mRNA expression levels. " $\mathrm{P}<0.01$ vs. control group; ${ }^{~} \mathrm{P}<0.01$ vs. anti-miR-146a group. TLR4, Toll-like receptor 4; miR, microRNA; iNOS, inducible nitric oxide synthase; COX-2, cyclooxygenase- 2 .

TLR4 inhibitor reduces the function of anti-miR-146a on iNOS and COX-2 mRNA expression within a model of ALI in $16 H B E$ cells. The increase of iNOS and COX-2 mRNA expression levels in the model of ALI induced by anti-miR-146a was significantly suppressed by the inhibition of TLR4, compared with the levels observed in the anti-miR-146a group (Fig. 10). MicroRNA-146a/TLR4 signaling protects against severe burn-induced remote ALI in rats via suppression of iNOS and COX-2 expression.

\section{Discussion}

Various mechanisms are involved in severe burn-induced remote ALI. Firstly, damage to inflammatory mediators, including proinflammatory cytokines and alexins, and lung tissues occurs. Secondly, the increase of cell adhesion molecule expression levels within neutrophils, granulocytes and endothelial cells following stimulation by proinflammatory cells occurs, and adhesive abilities increase (4). Subsequently, neutrophils and granulocytes invade the inflammatory lesions and, once activated, these cells undergo a respiratory burst, degranulation and release metabolites, including fibrinolysin, active oxygen and arachidonic acid. These molecules may damage VECs, tissues and other cells (13). Thirdly, the adhesion of WBCs and VECs, together with the accumulation of WBCs, may block capillaries,



Figure 11. miR-146a/TLR4 signaling protects against severe burn-induced remote acute lung injury in rats through anti-inflammation. miR, microRNA; TLR4, Toll-like receptor 4 ; NF- $\kappa \mathrm{B}$, nuclear factor- $\kappa \mathrm{B}$; iNOS, inducible nitric oxide synthase; COX-2, cyclooxygenase-2.

leading to thrombosis, thus resulting in reduced microcirculatory hemoperfusion (13). Consequently, a disproportional ratio 
of ventilation/blood flow may occur, inducing hypoxemia (14). In addition, the permeability of pulmonary vasculatures may increase, causing pulmonary edema. Fourth, the lung has the anatomical and physiological features of low tension, low resistance and high flow, and so circulatory inflammatory mediators may easily accumulate within the lung (4). In the present study, the expression of miR-146a was significantly downregulated in a burn-induced rat model of ALI compared with the levels in the control group and downregulation of miR-146a increased inflammation in a model of ALI. MiR-146a downregulation may worsen inflammation within the ALI model. Chen et al (15) indicated that miR-146a may regulate glucose-induced inflammation of the retina and kidney in diabetes or animal/in vitro models.

Endogenous nitric oxide (NO) is a free radical with high activity, and serves as a signaling molecule and a toxic molecule with diverse biological functions (4). Excessive or deficient endogenous NO may damage tissues and cells, and disrupt the equilibrium (4). It has been reported that iNOS itself possesses the activity of oxygen radicals to induce $\mathrm{O}_{2}$ production (16). NO levels may decrease once iNOS is inhibited, along with reduced $\mathrm{O}_{2}$ (4). In the present study, upregulated miR-146a expression levels were associated with decreased iNOS mRNA expression within the model of ALI. Furthermore, Li et al (17). suggested that miR-146a may reduce IL-6, IL-12, TNF- $\alpha$ and iNOS levels in systemic juvenile idiopathic arthritis.

Expression of inflammatory factors may be initiated and regulated following $\mathrm{NF}-\kappa \mathrm{B}$ activation (18). Epoxidase is an important rate-limiting enzyme during the synthesis of prostaglandin and has two isozymes, COX-1 and COX-2 (4). NF- $\kappa \mathrm{B}$ activation may induce COX-2 activity, resulting in increased levels of the immune-suppressor prostaglandin (18), leading to immunosuppression following infection (19). Therefore, following immunosuppression, the incidence rates of infection and mortality are high (18). To the best of our knowledge, the present study is the first to reveal that upregulated miR-146a may decrease COX-2 mRNA expression levels in a model of ALI. Sato et al (20) reported that miR-146a inhibition increased COX-2 expression in chronic obstructive pulmonary disease.

Severe burn-induced remote ALI is an infectious lung disease (21). It is predominantly caused by bacterial infection-induced septicopyemia (4). LPS, the primary element of gram-negative bacterium cytoderm, is the major pathogenic factor $(4,22)$. LPS may activate the signal transduction system via an interaction with a corresponding receptor. In addition, it may give rise to the activation of $N F-\kappa B$, initiating genetic transcription $(4,23)$. However, proinflammatory factors are produced to exert toxic functions $(4,23)$. TLR has been considered to be a notable receptor that mediates LPS signal transduction (4). In the present study, miR-146a notably suppressed TLR4/NF- $\kappa \mathrm{B}$ signaling within a model of ALI. Ye and Steinle (24) demonstrated that miR-146a may attenuate inflammation via TLR4/NF- $\kappa$ B and TNF- $\alpha$ within high glucose-induced primary human retinal microvascular endothelial cells. In the present study, TLR4/NF- $\kappa \mathrm{B}$ signaling was analyzed; therefore, further studies into the effects of miR-146a on NF- $\kappa \mathrm{B}$ signaling in ALI must be conducted.

In summary, miR-146a may protect against severe burn-induced remote ALI in rats. Specifically, miR-146a exerts anti-inflammatory effects via the TLR4/NF- $\mathrm{B}$ signaling pathway and inhibits iNOS and COX-2 expression (Fig. 11).
However, further investigation of miR-146 for the treatment of severe burn-induced remote ALI is required.

\section{Acknowledgements}

Not applicable.

\section{Funding}

No funding was received.

\section{Availability of data and materials}

The analyzed data sets generated during the study are available from the corresponding author on reasonable request.

\section{Authors' contributions}

JZ designed the experiment. JL and HY and XC performed the experiment. JZ analyzed the data and wrote the manuscript.

\section{Ethics approval and consent to participate}

All experimental protocols were undertaken in accordance with the Guide for the Care and Use of Laboratory Animals by the National Institutes of Health, with the approval of the Animal Experimental Ethics Committee of the First Affiliated Hospital of Nanchang University (Nanchang, China).

\section{Consent for publication}

Not applicable.

\section{Competing interests}

The authors declare that they have no competing interests.

\section{References}

1. Tianzhu Z and Shumin W: Esculin inhibits the inflammation of LPS-induced acute lung injury in mice via regulation of TLR/NF-kappaB pathways. Inflammation 38: 1529-1536, 2015.

2. Jiang S, Park DW, Tadie JM, Gregoire M, Deshane J, Pittet JF, Abraham E and Zmijewski J: Human resistin promotes neutrophil proinflammatory activation and neutrophil extracellular trap formation and increases severity of acute lung injury. J Immunol 192: 4795-4803, 2014.

3. Ferrando C, Aguilar G, Piqueras L, Soro M, Moreno J and Belda FJ: Sevoflurane, but not propofol, reduces the lung inflammatory response and improves oxygenation in an acute respiratory distress syndrome model: A randomised laboratory study. Eur J Anaesthesiol 30: 455-463, 2013.

4. Bai C, Li T, Sun Q, Xu T, Yu J, Wang Y and Wei L: Protective effect of baicalin against severe burn-induced remote acute lung injury in rats. Mol Med Rep 17: 2689-2694, 2018.

5. Fu PK, Wu CL, Tsai TH and Hsieh CL: Anti-inflammatory and anticoagulative effects of paeonol on LPS-induced acute lung injury in rats. Evid Based Complement Alternat Med 2012: 837513, 2012.

6. Zhang H, Sun T, Liu Z, Zhang J, Wang X and Liu J: Systemic inflammatory responses and lung injury following hip fracture surgery increases susceptibility to infection in aged rats. Mediators Inflamm 2013: 536435, 2013.

7. Shim DW, Han JW, Sun X, Jang CH, Koppula S, Kim TJ, Kang TB ansd Lee KH: Lysimachia clethroides Duby extract attenuates inflammatory response in Raw 264.7 macrophages stimulated with lipopolysaccharide and in acute lung injury mouse model. J Ethnopharmacol 150: 1007-1015, 2013. 
8. Fang Y, Gao F, Hao J and Liu Z: microRNA-1246 mediates lipopolysaccharide-induced pulmonary endothelial cell apoptosis and acute lung injury by targeting angiotensin-converting enzyme 2. Am J Transl Res 9: 1287-1296, 2017.

9. Cao Y, Lyu YI, Tang J and Li Y: MicroRNAs: Novel regulatory molecules in acute lung injury/acute respiratory distress syndrome. Biomed Rep 4: 523-527, 2016.

10. Rajasekaran S, Pattarayan D, Rajaguru P, Sudhakar Gandhi PS and Thimmulappa RK: MicroRNA regulation of acute lung injury and acute respiratory distress syndrome. J Cell Physiol 231: 2097-2106, 2016.

11. Bai X, Fan L, He T, Jia W, Yang L, Zhang J, Liu Y, Shi J, Su L and $\mathrm{Hu}$ D: SIRT1 protects rat lung tissue against severe burn-induced remote ALI by attenuating the apoptosis of PMVECs via p38 MAPK signaling. Sci Rep 5: 10277, 2015.

12. Livak KJ and Schmittgen TD: Analysis of relative gene expression data using real-time quantitative PCR and the 2(-Delta Delta C(T)) method. Methods 25: 402-408, 2001.

13. Jernigan PL, Hoehn RS, Blakeman TC, Heyl J, Robinson BR, Pritts TA and Branson RD: Portable mechanical ventilation with closed-loop control of inspired fraction of oxygen maintains oxygenation in the setting of hemorrhage and lung injury. J Trauma Acute Care Surg 79: 53-59, 2015.

14. Raghavendran K, Davidson BA, Huebschmann JC, Helinski JD, Hutson AD, Dayton MT, Notter RH and Knight PR: Superimposed gastric aspiration increases the severity of inflammation and permeability injury in a rat model of lung contusion. J Surg Res 155: 273-282, 2009.

15. Chen S, Feng B, Thomas AA and Chakrabarti S: miR-146a regulates glucose induced upregulation of inflammatory cytokines extracellular matrix proteins in the retina and kidney in diabetes. PLoS One 12: e0173918, 2017.

16. Li X, Liu Z, Jin H, Fan X, Yang X, Tang W, Yan J and Liang H: Agmatine protects against zymosan-induced acute lung injury in mice by inhibiting NF- $\kappa$ B-mediated inflammatory response Biomed Res Int 2014: 583736, 2014.
17. Li D, Duan M, Feng Y, Geng L, Li X and Zhang W: MiR-146a modulates macrophage polarization in systemic juvenile idiopathic arthritis by targeting INHBA. Mol Immunol 77: 205-212, 2016.

18. Quesada AE, Nguyen ND, Rios A and Brown RE: Morphoproteomics identifies constitutive activation of the mTORC2/Akt and NF- $\kappa$ B pathways and expressions of IGF-1R, Sirt1, COX-2, and FASN in peripher lymphomas: Pathogenetic implications and therapeutic options. Int J Clin Exp Pathol 7: 8732-8739, 2014.

19. Kang SS, Cuendet M, Endringer DC, Croy VL, Pezzuto JM and Lipton MA: Synthesis and biological evaluation of a library of resveratrol analogues as inhibitors of COX-1, COX-2 and NF-kappaB. Bioorg Med Chem 17: 1044-1054, 2009.

20. Sato T, Liu X, Nelson A, Nakanishi M, Kanaji N, Wang X, Kim M, Li Y, Sun J, Michalski J, et al: Reduced miR-146a increases prostaglandin $\mathrm{E}_{2}$ in chronic obstructive pulmonary disease fibroblasts. Am J Respir Crit Care Med 182: 1020-1029, 2010.

21. Yang H, Li Y, Huo P, Li XO, Kong D, Mu W, Fang W, Li L, Liu N, Fang L, et al: Protective effect of Jolkinolide B on LPS-induced mouse acute lung injury. Int Immunopharmacol 26: 119-124, 2015.

22. Kim HJ, Lee HS, Chong YH and Kang JL: p38 Mitogen-activated protein kinase up-regulates LPS-induced NF-kappaB activation in the development of lung injury and RAW 264.7 macrophages. Toxicology 225: 36-47, 2006.

23. Wang G, Huang X, Li Y, Guo K, Ning P and Zhang Y: PARP-1 inhibitor, DPQ, attenuates LPS-induced acute lung injury through inhibiting NF- $\mathrm{BB}$-mediated inflammatory response. PLoS One 8: e79757, 2013.

24. Ye EA and Steinle JJ: miR-146a attenuates inflammatory pathways mediated by $\mathrm{TLR} 4 / \mathrm{NF}-\kappa \mathrm{B}$ and $\mathrm{TNF} \alpha$ to protect primary human retinal microvascular endothelial cells grown in high glucose. Mediators Inflamm 2016: Article ID 3958453, 2016. 